\title{
Mutations at Codon 641 of Exon 16 of EZH2 in Circulating Tumor DNA: A Biomarker for Relapse in Patients With Diffuse Large B-cell Lymphoma Treated With the R-CHOP Scheme.
}

\section{José Díaz-Chávez}

Instituto Nacional de Cancerología: Instituto Nacional de Cancerologia

Olga Gutiérrez-Hernández

Instituto Nacional de Cancerologia

Lucia Taja-Chayeb

Instituto Nacional de Cancerologia

Sindy Gutiérrez-Chavarría

Instituto Nacional de Cancerologia

Alejandro Aviles-Salas

Instituto Nacional de Cancerologia

Myma Candelaria ( $\nabla$ candelariahmgloria@gmail.com )

Instituto Nacional de Cancerologia https://orcid.org/0000-0002-5478-714X

\section{Research Article}

Keywords: EZH2, ctDNA, lymphoma, prognosis

Posted Date: January 7th, 2022

DOl: https://doi.org/10.21203/rs.3.rs-1190066/v1

License: (c) (i) This work is licensed under a Creative Commons Attribution 4.0 International License.

Read Full License 


\section{Abstract}

Background: The epigenetic regulator EZH2 is a subunit of the polycomb repressive complex 2 (PRC2), methylates H3K27, resulting in transcriptional silencing. The mutation at $\mathrm{Y} 646$ amino acid in the EZH2 gene is mutated in up to $40 \%$ of B-cell lymphomas.

Methods: We compared the presence of exon 16 EZH2 mutations in tumor samples and ctDNA in a prospective trial. The mutations were determined by sanger sequencing, and by ddPCR. We also evaluated the impact of these mutations on response, relapse, and survival.

Results: One hundred and thirty-eight cases were included. Ninety-eight were germinal center, and twenty had EZH2 mutations. Mean follow-up (IQR 25-75) was 23 (7- 42) months. The tumor samples were considered the standard of reference. Considering the results of the mutation in ctDNA by Sanger sequencing, the sensibility (Se) and specificity (Sp) were $52 \%$ and $99 \%$, respectively. After adding the ddPCR analysis, the Se and Sp increased to 95 and $100 \%$, respectively. After bivariate analysis, only the presence of double-hit lymphoma ( $p=0.04)$, or $E Z H 2$ mutations were associated with relapse. The median PFS (95\% Interval confidence) was 27.7 (95\% IC: 14-40) vs 44.1 (95\% IC: 40-47.6) months for the mutated vs wt patients.

Conclusions: The ctDNA is usefull to analyse $E Z H 2$ mutations, which have an impact in PFS.

\section{Introduction}

Diffuse Large B Cell Lymphoma (DLBCL) constitutes the most common of all aggressive types of lymphomas [1]. It is a clinically and molecularly heterogeneous malignant lymphoproliferative disease [2, 3]. Traditionally, it has been classified into morphological variants, molecular subtypes, and distinct disease entities. Among no-otherwise-specified (NOS) cases, an accepted grouping is either germinal center (GC) subtype and or non-germinal center (non-GC) subtype. GC subtype has a significantly better prognosis. However, within the GC-subtype, some patients show Myc rearrangement with co-expression of BCL2 or BCL6, defined as double or triple hit lymphomas [2)] which have a more aggressive clinical behavior. Recently, the presence of $E Z H 2$ mutations has also been implicated in the prognosis of DLBCL [4-6].

The epigenetic regulator $\mathrm{EZH} 2$ is a subunit of the polycomb repressive complex 2 (PRC2), methylates $\mathrm{H} 3 \mathrm{~K} 27$, resulting in transcriptional silencing [7-8]. The overexpression of EZH2 has been identified as a driver in lymphomagenesis [9]. In addition, the mutation at $Y 646$ amino acid in the $E Z H 2$ gene is recurrently and significantly mutated in up to $40 \%$ of B-cell lymphomas [10], and particularly in approximately $13-22 \%$ of DLBCL [4, 11-12]. The mutations of Tyr641 (Y641F, Y641N, Y641S, and Y641H) are deficient catalysts of unmodified H3K27 methylation relative to the wild type (wt) enzyme. However, these mutants are superior to the wt enzyme in catalyzing further methylation to mono, and especially the demethylated H3K27 peptides $[13,14]$. The final effect of these mutations is gene silencing. 
Traditionally, mutations are documented in tumor samples. However, with the development of new techniques, the identification of circulating tumor DNA (ctDNA) is available and useful to monitor tumorspecific molecules in the blood, with a sensitivity approaching $1 \times 10^{6}$ cells [15] and also with high tumor specificity. Also, the access to serial blood samples allows monitoring these tumor-specific changes and follow-up during treatment [16]. This quantitative approach has been used as a marker for the identification of tumor biology, and to predict long-term outcome [17].

Although ctDNA promises as a monitoring tool, the standardization of the collection and processing is necessary to improve DNA preservation and facilitate accurate testing and interpretation of the results. Therefore, we analyzed the feasibility to determine the presence of exon 16 EZH2 mutations in ctDNA, when compared with the tumor samples as the standard of reference. In the same direction, we also evaluated the clinical impact of these mutations in terms of response, relapse and survival in a cohort of patients with DLBCL.

\section{Material And Methods}

This is a prospective cohort, non interventional study, including consecutive patients diagnosed with DLBCL, who were attended at the National Cancer Institute (Mexico City, Mexico) between January 2017 till December 2019. The last follow-up was on July 31st 2021. The inclusion criteria were: age older than 18 years, histopathological diagnosis of DLBCL, without previous treatment and candidate to be treated with RCHOP. We excluded patients with hepatitis B or C or HIV, as well as those receiving any other treatment regimen.

Clinical variables analyzed were: Age, International Prognostic Index (IPI), presence of B symptoms, bulky disease, clinical-stage by Lugano classification [18], serum albumin, lactate dehydrogenase levels (LDH), and Beta2-microglobulin levels, and performance status determined by the Eastern Cooperative Oncology Group (ECOG) score [19]. Histopathologic variables were GC versus no-GC by Hans nomogram [20], as well as BCL2, BLC6, and MYC expression, and double-hit lymphoma. Briefly, the GC type was defined by expression of CD10, or CD10 (-), BCL6 (+), MUM1 (-). Non-GC type was considered if CD10 (-), BCL6 (-), or CD10 (-), BCL6 (+), MUM1 (+). The cut-off value to consider BCL2 positive was $50 \%$, but $30 \%$ for BCL6 and $40 \%$ for MYC expression. Lymphomas co-expressing MYC and BCL2 or BLC6 were considered double-expressors. Double-hit lymphomas were defined as those with at least $10 \%$ of MYC rearrangements were present by fluorescence in situ hybridization (FISH) as previously described [21]. Regarding $E Z H 2$ status, they were classified as mutated or wild type.

All patients were treated with 6 cycles of RCHOP regimen: IV rituximab, $375 \mathrm{mg} / \mathrm{m} 2$ on day 1 ; IV cyclophosphamide, $750 \mathrm{mg} / \mathrm{m} 2$ on day 1 ; IV doxorubicin, $50 \mathrm{mg} / \mathrm{m} 2$ on day $1 ;$ IV vincristine, $1.4 \mathrm{mg} / \mathrm{m} 2$, with capping at $2 \mathrm{mg}$, on day 1 ; and oral prednisone, $100 \mathrm{mg}$ daily on days $1-5 .{ }^{18}$ Fluoro-deoxyglucose PET-CT positron emission tomography combined with computer tomography (PET-CT) was done at diagnosis, and at the end of treatment. 
Analyzed outcomes were clinical response after chemotherapy, risk of relapse, Progression-free survival (PFS), and overall survival (OS). All patients signed informed consent.

\section{Samples.}

Tumor samples: 4 sections of the tumor area of 8 to $10 \mu \mathrm{m}$ from each patient were used. FFPE samples were processed with the AllPrep ${ }^{\circledR}$ DNA/RNA FFPE Kit Cat. No. 80234 (Qiagen, Germany) according to the manufacturer's instructions. DNA was quantified with nanodrop and stored at $-20^{\circ} \mathrm{C}$.

Peripheral blood: $2 \mathrm{~mL}$ of plasma was used, as specified by the manufacturer's instructions. The ctDNA was extracted using QIAamp Circulating Nucleic Acid Kit (50) Cat. No. 55114 (Qiagen, Germany). The amount of extracted ctDNA was quantified with Qubit Fluorometer using Qubit ${ }^{\text {TM }}$ dsDNA HS Assay Kit Cat. No. Q32851 (Thermo Fisher Scientific) following the manufacturer's instructions.

PCR amplification. PCR reactions were performed in a total volume of $25 \mu \mathrm{l}$ containing $50 \mathrm{ng}$ of DNA, 1 $\mu \mathrm{mol} / \mathrm{L}$ of each primer (forward: 5'-ATCTATTGCTGGCACCATCT-3' and reverse: $5^{\prime}$ CCAATCAAACCCACAGACTTAC-3'), $200 \mu \mathrm{mol} / \mathrm{L}$ dNTPs (Applied Biosystems, Foster City, CA), $2 \mathrm{mM} \mathrm{MgCl}{ }_{2}$, $0.25 \mathrm{U}$ TaqPol (Applied Biosystems, Foster City, CA) and buffer 1X provided by the manufacturer. PCRs were performed in a 2700 Thermalcycler (Applied Biosystems). The amplifications were done as follows: initial denaturation at $95^{\circ} \mathrm{C}$ for $5 \mathrm{~min}$ and a final extension at $72^{\circ} \mathrm{C}$ for $5 \mathrm{~min}$; denaturation at $95^{\circ} \mathrm{C}$ for 30 seconds, annealing for 30 seconds at $58^{\circ} \mathrm{C}$ and extension was done at $72^{\circ} \mathrm{C}$ for 30 seconds for 40 cycles. Amplification was verified by gel electrophoresis.

Sanger sequencing.

The PCR products were sequenced in at least two independent amplification reactions to analyze the presence of mutations in Exon 16 of EZH2, using the Reverse primer: 5'-CCAATCAAACCCACAGACTTAC3'(Integrated DNA Technologies; Standard desalted purification synthesis) PCR amplicons were purified using isopropanol precipitation. According to the manufacturer's instructions, the purified DNA was diluted and cycle-sequenced using the ABI BigDye Terminator kit v3.1 (ABI, Foster City, CA, USA). Sequencing reactions were electrophoresed in an ABI3500 genetic analyzer. Electropherograms were analyzed, and the sequences obtained were compared with the $E Z H 2$ reference sequence (GenBank NG_032043.1).

Genomic DNA from the DLBCL cell lines SU-DHL-6, SU-DHL-10, and Pfeiffer were used as controls for the EZH2 Y641N, Y641F mutations, and wt, respectively.

Droplet digital PCR (ddPCR).

Those samples of ctDNA that showed a discordant result after Sanger sequencing were analyzed by ddPCR. The tumor DNA was considered the standard of reference. 
We amplified 75 bp of EZH2 (exon 16) using primers 5'-TGAATACAGGTTATCAGTGC-3' and 5'TCAAAGATCCTGTGCAGA-3' (Integrated DNA Technologies; Standard desalted purification synthesis) and used Custom PrimeTime(C) Mini LNA probes (Integrated DNA Technologies) utilized for each of the four most commonly found somatic mutations at this hotspot: $\mathrm{Y} 641 \mathrm{H}\left(5^{\prime}-/ 56-\right.$

FAM/AGAA+CA+CT+GTGGAGAGGTA/3IABkFQ/-3'); Y641N

(5'-/5HEX/AGAA+AA+CTGT+GGA+GAGGTA/3IABkFQ/-3'); Y641F(5'-/56-

FAM/AGAAT+TCT+GTG+GAG+AGGTA/3IABkFQ/-3'; Y641S(5'-/56-

FAM/AGAA+TCCTGT+GGA+GAGGTA/3IABkFQ/-3') and also one probe targeting the wild-type allele (5'-/56-6-FAM/AGAA+TACTG+TGGA+GAGGTA/3IABkFQ/-3'; + states for Locked Nucleic Acid or LNA bases), these probes were previously designed and reported [22].

The reaction mixture for ddPCR contained $10 \mu \mathrm{g}$ of ctDNA, $250 \mathrm{nmol} / \mathrm{L}$ forward and reverse primers, 250 nmol/L FAM-labeled wt probe, 250 nmol/L HEX-labeled Y641N, 250 nmol/L FAM-labeled Y641S, Y641H, Y641F probe, and $11 \mu \mathrm{l}$ of $2 \times$ ddPCR Supermix for Probes (BioRad Laboratories, Pleasanton, CA, USA). Distilled water was added to achieve a final volume of $22 \mu \mathrm{l}$. The reaction mixture was then partitioned into nanoliter-sized droplets using QX200 Droplet Generator TM (BioRad Laboratories), in which the target DNA was randomly distributed into the droplets. Then, the droplets were transferred to a 96-well plate for PCR reaction in a thermal cycler (Biorad). The PCR program was initiated and held at $95^{\circ} \mathrm{C}$ for $10 \mathrm{~min}$, followed by 39 cycles at $94^{\circ} \mathrm{C}$ for $30 \mathrm{sec} 58^{\circ} \mathrm{C}$ for $1 \mathrm{~min}$, and $98^{\circ} \mathrm{C}$ for $10 \mathrm{~min}$. The PCR product from each well was then subjected to the QX200 Droplet Reader (BioRad Technologies), which analyzed the fluorescence of each droplet individually using a two-color detection system. Custom software (QuantaSoft; BioRad Technologies) was used to count the number of droplets within each gate.

\section{Statistical analysis.}

A descriptive analysis was done for demographic and clinical characteristics. Median and its interquartile range (IQR) was used as a measure of dispersion. Clinical and histological variables were compared between wt and mutated cases by Chi-squared test, and T-student's test, as required. Response was evaluated by Lugano criteria (18). Progression-free survival (PFS) was defined, from the date of diagnosis, until relapse, progression, or the last visit. Overall survival (OS) was defined from the diagnosis date until death or last visit.

ctDNA concentrations were measured and compared by bivariate analysis with the following clinical prognostic factors: LDH levels, clinical stage, IPI score, response to treatment, and presence of relapse.

Results of $E Z H 2$ mutations in the tumor sample and ctDNA were compared, considering the tumor sample as the standard of reference. Sensitivity (Se), specificity (Sp), positive predictive value (PPV), as well as negative predictive value (NPV) were calculated. Se was calculated with true positive/ (true positive + false negative). Sp was calculated with: True negative / (true negative + false positive). PPV was: True positive/ (true positive+ false positive), and NVP was obtained with: true negative/ (false negative + true negative). 
The Kaplan-Meier method was used to construct survival curves, and the Log-rank test was used for comparisons. The survival curves compared the mutated and wt cases.

The proportionality assumptions and interaction terms were checked in the final models. The SPSS version 23 software (IBM, Corp. Armonk, NY) was used for computations.

\section{Results}

\section{Patients}

A total of 138 cases were included, most of cases were male $(n=74,53.6 \%)$, with at least one site of extranodal involvement $(n=87,63 \%)$, and an advanced disease $(n=96,69.5 \%)$. The median (25-75 IQR) age was 60.1 (50.75-70) years. According to Hans nomogram, most were classified as germinal center (GC) $(n=98,71 \%)$. Clinical and tumor characteristics are detailed in Table 1. From all clinical and histological variables, only the presence of advanced clinical stage was statistically significant different between wt and mutated cases. All mutated patients belonged to the GC cell of origin. All patients were treated with six cycles of R-CHOP. 
Table 1

Clinical and histological characteristics at diagnosis.

\begin{tabular}{|llll|}
\hline & $\mathbf{n}(\%)$ & $\mathbf{n}(\%)$ & $\mathbf{P}$ \\
& wt & Mutated & \\
\hline Total & $118(85.5)$ & $20(14.5)$ & - \\
\hline Gender & $52(37.7)$ & $12(8.7)$ & 0.18 \\
Memale & $66(47.8)$ & $8(5.8)$ & \\
\hline Mean age & & & \\
(25-75 IQR) & 60.46 & 58.55 & 0.68 \\
ECOG* & $(50-71)$ & $(52.5-66)$ & \\
0-1 & $86(62.3)$ & $12(8.7)$ & 0.097 \\
$>2$ & $32(23.2)$ & $8(5.8)$ & \\
\hline Presence of B symptoms & $51(37)$ & $12(8.7)$ & 0.164 \\
Yes & $67(48.5)$ & $8(5.8)$ & \\
No & & & \\
\hline Presence of Bulky disease & $43(31.2)$ & $11(8.0)$ & 0.076 \\
Yes & $76(55.0)$ & $8(5.8)$ & \\
No & & & \\
\hline Clinical stage & & & \\
I- II & 40 & 18 & \\
III- IV & & & \\
\hline
\end{tabular}

*ECOG: Eastern Cooperative Oncology Group
+IPI: International Prognostic Index
++GC: Germinal-center
+++Non-GC: Non-germinal center

Figure legends. 


\begin{tabular}{|llll|}
\hline & $\mathbf{n}(\%)$ & $\mathbf{n}(\%)$ & $\mathbf{P}$ \\
& wt & Mutated & \\
\hline IPI score+ & $38(27.5)$ & $3(2.2)$ & 0.38 \\
Low & $14(10.1)$ & $2(1.5)$ & \\
Intermediate-low & $17(12.3)$ & $3(2.2)$ & \\
Intermediate-high & $49(35.5)$ & $12(8.7)$ & \\
High & & & \\
\hline Cell of origin & $78(56.5)$ & $20(14.5)$ & 0.008 \\
GC++ & $40(29.0)$ & 0 & \\
\hline Non-GC+++ & & & \\
\hline Double hit & $12(8.7)$ & $3(2.2)$ & 0.52 \\
\hline Extranodal sites & 97 & 11 & 0.33 \\
\hline 0 - 1 & 21 & 9 & \\
\hline$\geq 2$ & & & \\
\hline B 2 microglobulin & $39(28.3)$ & $6(4.4)$ & \\
\hline Normal & $79(57.2)$ & $14(10.1)$ & \\
increased & & & \\
\hline *ECOG: Eastern Cooperative Oncology Group & \\
\hline +IPI: International Prognostic Index & & \\
\hline++ GC: Germinal-center & & & \\
\hline+++ Non-GC: Non-germinal center & & \\
\hline Figure legends. & & & \\
\hline
\end{tabular}

\section{Samples}

All cases had tumor and peripheral blood samples and were analyzed for mutations at codon 641 in exon 16 of $E Z H 2$.

Tumor samples: Twenty patients of 138 cases with DLBCL (14.5\%), corresponding to $20.4 \%$ of the $98 \mathrm{GC}$ DLBCL had EZH2 mutations, as follows: Y641F [n=7, 5.1\%], Y641N [n=4, 2.9\%], Y641H [n=3, 2.2\%], Y641S [n=3, 2.2\%], I638T [n=1,0.7\%]. Two patients had a double mutation: $Y 641 \mathrm{~N}+\mathrm{F} 637 \mathrm{~L}[\mathrm{n}=1,0.7 \%]$, $\mathrm{Y} 641 \mathrm{~S}+\mathrm{Y} 641 \mathrm{~F}[\mathrm{n}=1,0.7 \%]$. 
The median concentration (IQR 25-75) of ctDNA was $1555.63 \mathrm{ng} / \mathrm{mL}(904.0$ - 4410.0). An association was documented between median (IQR 25-75) ctDNA and LDH values: $1085 \mathrm{ng} / \mathrm{mL}$ (659-1767) vs 1920 $\mathrm{ng} / \mathrm{mL}(952-5860)$ in patients with normal vs elevated values of $\mathrm{LDH}$, respectively $(\mathrm{p}=0.01)$. No association was documented within IPI score, clinical stage disease, response rate or relapse rate, and ctDNA concentration.

Regarding ctDNA analysis, after initial Sanger sequencing, only eleven mutations were documented: Y641F [n=4, 2.9\%], Y641N [n=2, 1.4\%], Y641H [n=2, 1.4\%], Y641S [n=1, 0.7\%\%], Y641C [n=1, 0.7\%], Y641N $+F 637 L[n=1,0.7 \%]$. The figure 1 shows an example of these chromatograms. Those patients with a mutation in the tumor, but wt by ctDNA in plasma by Sanger sequencing analysis, were considered false negatives, and subsequently were evaluated by ddPCR. After this analysis, nine mutations (previously documented in tumor samples) were confirmed: Y641F [n=3, 2.1\%], Y641N [n=2, 1.4\%], Y $641 \mathrm{H}[\mathrm{n}=1,0.7 \%]$, Y641S [n=2, 1.4\%], Y641S + Y641F [n=1,0.7\%]. Considering the mutations results in ctDNA by Sanger sequencing, the Se and Sp were $52 \%$ and $99 \%$, respectively. After adding the ddPCR analysis, the Se and Sp increased to 95 and $100 \%$, respectively. Predictive values are detailed in figure 2 .

\section{Clinical response}

Most of patients achieved complete $(70 \%)$ or partial $(14 \%)$ response. Only eleven (16\%) progressed during treatment. By bivariate analysis, CR was associated with the presence of bulky disease $(p=0.033)$, Lugano clinical stage $(p=0.024)$, and IPI score $(p=0.03)$. Neither the ctDNA concentration or the presence of $E Z H 2$ mutations was associated with response. After multivariate analysis, only clinical stage remained significant (OR 1.98; 95\% IC 1.122-3.378, $p=0.02)$ for response.

Relapse and survival: Mean follow-up (IQR 25-75) was 23 (7- 42) months. During this time, twenty-four patients $(17.4 \%)$ relapsed. After bivariate analysis, only the presence of double-hit lymphoma $(p=0.04)$, EZH2 mutations either on tumor analysis (0.047) or ctDNA (0.021) were factors associated with relapse. Median (IQR 25-75) PFS was 22.6 (6-40.2) months. The Kaplan-Meier PFS curves of mutated and wt patients in tumor and ctDNA analysis are displayed in figure 3. The median PFS (95\% Interval confidence) was 27.7 (95\% IC: 14-40) vs 44.1 (95\% IC: $40-47.6)$ months in the EZH2 mutated vs wt patients, respectively.

The median (95\% IC) OS was not different between the mutated or wt EZH2 patients: 40.24 (95\% IC: 30.7$49.76)$ vs 42.43 (95\% IC: $38.67-46.18)$, respectively.

\section{Discussion}

The detection of somatic mutations directly from ctDNA is an attractive alternative, because ctDNA is a non-invasive, real time, tumor-specific biomarker, an alternative source of tumor DNA for genotyping purposes. However, the molecular aberrations within lymphomas are heterogeneous and different methods are employed for such purposes. In this cohort, the addition of ddPCR analysis to Sanger sequencing increased the sensitivity from 52 to $95 \%$, with a PPV and of $100 \%$ and $92 \%$, respectively. 
In healthy subjects the cell-free DNA (cfDNA) derives from the apoptosis of hematopoietic cells. In lymphoma patients, the total amount of cfDNA has a median concentration of $30 \mathrm{ng} / \mathrm{mL}$ of plasma [2324]. The normal cfDNA requires to be discriminated from ctDNA, and the test used for ctDNA detection and quantification requires to suppress the technical and biological noise in order to achieve the required sensitivity and specificity.

The ctDNA concentrations vary among the lymphoma subtypes, with higher values in Diffuse large B cell lymphoma, Hodgkin lymphoma and mantle cell lymphoma, and lower levels in low-grade B lymphomas, as follicular lymphoma. [16, 25-28]. In this cohort, the median ctDNA concentrations were within the reported range. Some studies [29-31] have concluded that higher levels of ctDNA are associated with different prognostic markers, as advanced clinical stage, poor risk prognostic categories evaluated with IPI score, or survival. However, in this cohort we only found an association between ctDNA and elevated levels of LDH, none other prognostic parameter, as clinical stage, or IPI correlated with ctDNA concentrations. This difference may be related to the fact that we had very few patients with low clinical stages by low grade, according to IPI score, when compared with other authors. In the same direction, we could not demonstrate a correlation with ctDNA levels and response to treatment, or survival since we collected only a sample at diagnosis; however other authors [30-33], have indeed demonstrated a correlation between the ctDNA levels and a worse response to treatment, or with survival [30, 34-35], evaluating the kinetics of ctDNA.

It has been proposed, that the liquid biopsy can inform about the whole intratumor heterogeneity. The concordance of results between the analysis in tumor samples, in comparison with ctDNA may vary with the proportion of a mutation within tumoral tissue and in ctDNA. In this study, the analysis by Sanger sequencing had a very low $\mathrm{Se}(52 \%)$. However, the addition of a more sensible technique, as ddPCR increased the detection of $E Z H 2$ mutations in all patients analyzed by this technique.

Ultra-deep generation sequencing (NGS) methodologies can identify a range of genetic alterations. For example, The Cancer Personalized Profile by Deep Sequencing (CAPP-Seq) is considered a diseasespecific selector, covering a set of exonic and intronic regions of known recurrent mutations for a specific cancer setting $[25,36]$. The CAPP-Seq relies on an updatable gene panel containing lymphoma genes, as well as somatic mutations; unfortunately, none commercial kits are available. Also, ddPCR assays are used to detect mutations but may not be representative of a fraction of ctDNA, unless a targeted mutation is known to be trunk in all lymphoma cases. In this sense, genotyping of ctDNA by CAPP-Seq allows the recovery of $100 \%$ of the tumor confirmed actionable mutations of DLBCL, as EZH2, MYD88, $C D 79 B[23,32]$.

In addition, ddPCR also measures absolute quantities by counting nucleic acid molecules encapsulated in defined water-in-oil-droplet partitions [25], and the reported sensitivity to detect XPO1, E57K, EZH2 Y641N, MYD88 and L265P mutations ranges from $80-100 \%$, with a $100 \%$ specificity $[27,37]$.

Camus et al. [37] documented the usefulness of ddPCR to quantify recurrent and potentially somatic mutations in ctDNA from 88 patients with DLBCL, including EZH2Y641 mutations. In addition, this 
author found a $100 \%$ concordance for somatic mutation detection between ddPCR and NGS. In our study, the sensitivity of this approach was $95 \%$, with a $100 \%$ specificity, and as has been described [37], no false-positive cases have been documented with this method. Dubois et al. [38] documented initially $22 \%$, and thereafter up to $24 \%$ frequency of $E Z H 2$ Y 641 mutations in GC- DLBCL, which is slightly higher than initially reported by Morin et al. $(\mathrm{n}=18 / 83,21.7 \%)$ [39]. In our study, we found a similar frequency of $E Z H 2$ Y641 mutations $(n=20 / 98,20.4 \%)$ in the same population.

Different authors have evaluated the clinical impact of EZH2 mutations in DLBCL in tumor samples [11, $16,29,40-41]$. However, recently, only Nagy et al. [42] has used liquid biopsy to evaluate the clinical role of $E Z H 2$ mutations by ddPCR, but in patients with follicular lymphoma, and correlated the variant allele frequency with the volume of metabolically active tumor sites observed on 18F-fluorodeoxyglucose positron emission tomography combined with computer tomography (PET-CT) scans. To our knowledge, this is the first study analyzing the $E Z H 2$ mutation using ctDNA to evaluate the frequency and the negative impact on PFS in Diffuse large B cell lymphoma. Furthermore, it will be interesting to determine other actionable mutations of DLBCL, as MYD88 and CD79B together EZH2 mutations in ctDNA, and analyze their impact in response to therapy and other clinical variables.

A personalized approach to cancer diagnosis implies integral tumor profiling for each patient, which might be possible by tracking plasma ctDNA tumor-related mutations. The purpose of studying biopsy specimens may be the selection of a personalized anticancer therapy, relevant to the mutational profile of the specific tumor. But, on the other hand, application of the plasma ctDNA analysis allows for the monitoring of disease dynamics and the prescribed therapy effectiveness in order to detect any residual tumor after resection, relapse, or even metastasis within a particular patient [43].

\section{Conclusions}

In conclusion, our data support the implementation in the clinic of the analysis of recurrent somatic mutations like $E Z H 2$ in ctDNA to diagnose early detection of molecular relapse, guide salvage therapy based on molecular targets, and identify molecular resistance mechanisms.

\section{Declarations}

Ethical approval and consent to participate: This trial was approved by IRB (register number CEI/966/15). All patients signed informed consent.

\section{Availability of supporting data.}

The corresponding author will make available the database of this study by request.

\section{Competing interest.}

All authors declare none conflict of interest. 


\section{Funding.}

This research was supported by the pharmacogenetics Laboratory from the Instituto Nacional de Cancerología.

The authors declare that no funds, grants, or other support were received during the preparation of this manuscript."

\section{Competing Interests.}

The authors have no relevant finantial or non-finaltial interests to disclose

\section{Author's contribution.}

J D-Ch. Sample analysis, analyzed results. Review manuscript

O G-H. Sample analysis, analyzed results. Review manuscript

L T-Ch Sample analysis, analyzed results. Review manuscript

S G-Ch. Clinical follow-up of patients. Review manuscript

A A-S:Pathological analysis of samples. Review manuscript

M. C: wrote the protocol, treated patients, clinical follow-up of patients, analyzed results, wrote manuscript.

\section{Ethics approval.}

This study was approved by the IRB (register number CEI/966/15). All patients signed informed consent.

\section{Author's information:}

Not applicable.

\section{References}

1. Horwitz SM, Zelenetz AD, Gordon LI, et al. Non-Hodgkin's Lymphomas, Version 3.2016. J Natl Compr Canc Netw. 2016;14(9):1067-79.

2. Nguyen L, Papenhausen P, Shao H. The Role of C-MYC in B-Cell Lymphomas: Diagnostic and Molecular Aspects. Genes. 2017;8(4):1-23.

3. Wu FT, Lu L, Xu W, Li JY. Circulating tumor DNA: clinical roles in diffuse large B-cell lymphoma. Ann Hematol. 2019;98(2):255-69.

4. Singh K, Gollapudi S, Mittal S, Small C, Kumar J, Ohgami RS. Point Mutation Specific Antibodies in BCell and T-Cell Lymphomas and Leukemias: Targeting IDH2, KRAS, BRAF and Other Biomarkers 
RHOA, IRF8, MYD88, ID3, NRAS, SF3B1 and EZH2. Diagnostics. 2021;11(4):600.

5. Chapuy B, Stewart Ch, Dunford AJ, et al. Molecular subtypes of diffuse large B cell lymphoma are associated with distinct pathogenic mechanisms and outcomes. Nat Med. 2018;24(5):679-90.

6. Schmitz R, Wright GW, Huang DW, et al. Genetics and Pathogenesis of Diffuse Large B-Cell Lymphoma. N Engl J Med. 2018;378(15):1396-407.

7. Müller J, Hart CM, Francis NJ, et al. Histone methyltransferase activity of a Drosophila Polycomb group repressor complex. Cell. 2002;111(2):197-208.

8. Czermin B, Melfi R, McCabe D, Seitz V, Imhof A, Pirrotta V. Drosophila enhancer of Zeste/ESC complexes have a histone $\mathrm{H} 3$ methyltransferase activity that marks chromosomal Polycomb sites. Cell. 2002;111(2):185-96.

9. Martin-Subero Jl, Kreuz M, Bibikova M, et al. New insights into the biology and origin of mature aggressive B-cell lymphomas by combined epigenomic, genomic, and transcriptional profiling. Blood. 2009;113(11):2488-97.

10. Bödör C, O’Riain C, Wrench D, Matthews J, lyengar S, Tayyib H, et al. EZH2 Y641 mutations in follicular lymphoma. Leukemia. 2011;25(4):726-9.

11. Oñate-Ocaña LF, Ponce-Martínez M, Taja-Chayeb $L$, et al. A Cohort Study of the Prognostic Impact of Exon-16 EZH2 Mutations in a Mexican-Mestizo Population of Patients with Diffuse Large B-Cell Lymphoma. Rev Invest Clin. 2021. May 27.

12. Yap DB, Chu J, Berg T, Schapira M, et al. Somatic mutations at EZH2 Y641 act dominantly through a mechanism of selectively altered PRC2 catalytic activity, to increase H3K27 trimethylation. Blood. 2011;117(8):2451-9.

13. Wigle T, Knutson SK, Jin L, Kuntz KW, Pollock RM, Richon VM. The Y641 mutation of EZH2 alters substrate specificity for histone H3 lysine 27 methylation states. FEBS lett. 2011;585(19):3011-4.

14. Sneeringer CJ, Scott MP, Kuntz KW, et al. Coordinated activities of wild-type plus mutant EZH2 drive tumor-associated hypertrimethylation of lysine 27 on histone H3 (H3K27) in human B-cell lymphomas. Proc Natl Acad Sci USA. 2010;107(49):20980-5.

15. Melani Ch, Wilson W, Roschewski M. Monitoring clinical outcomes in aggressive B-cell lymphoma: From imaging studies to circulating tumor DNA. Best Pract Res Clin Haematol. 2018;31(3):285-92.

16. Arzuaga-Mendez J, Prieto-Fernandez E, Lopez-Lopez E, Martin-Guerrero I, García-Ruiz JC, García Orad A. Cell-free DNA as a biomarker in diffuse large B-cell lymphoma: A systematic review. Crit Rev Oncol Hematol. 2019;139:7-15.

17. Kallam A, Adusumalli J, Fu K, Armitage JO. Circulating tumor DNA in lymphomas: Era of precision medicine. Adv in cell and gene therapy. 2020. https://doi.org/10.1002/acg2.81.

18. Cheson BD, Fisher RI, Barrington SF, et al. Recommendations for initial evaluation, staging, and response assessment of Hodgkin and non-Hodgkin lymphoma: the Lugano classification. J Clin Oncol. 2014;32(27):3059-68. 
19. Oken MM, Creech RH, Tormey DC, et al. Toxicity and response criteria of the Eastern Cooperative Oncology Group. Am J Clin Oncol. 1982;5(6):649-55.

20. Hans CP, Weisenburger DD, Greiner TC, et al. Confirmation of the molecular classification of diffuse large B-cell lymphoma by immunohistochemistry using a tissue microarray. Blood. 2004;103(1):275-82.

21. Solis-Armenta R, Cacho-Díaz B, Gutiérrez-Hernández O, Candelaria-Hernández M. Central Nervous System International Prognostic Index Impacts Overall Survival in Diffuse Large B-Cell Lymphoma Treated with R-Chop in a third Level Cancer Center from Mexico: A Survey of 642 patients. Rev Invest Clin. 2021;73(4):231-7.

22. Alcaide M, Yu S, Bushell K, et al. Multiplex Droplet Digital PCR Quantification of Recurrent Somatic Mutations in Diffuse Large B-Cell and Follicular Lymphoma. Clinical Chem. 2016;62(9):1238-47.

23. Rossi D, Spina V, Bruscaggin A, Gaidano G. Liquid biopsy in lymphoma. Haematologica. 2019;104(4):648-52.

24. Roschewski M, Dunleavy K, Pittaluga $S$, et al. Circulating tumour DNA and CT monitoring in patients with untreated diffuse large B-cell lymphoma: a correlative biomarker study. Lancet Oncol. 2015;16(5):541-9.

25. Condoluci A, Ross D. The future of cell-free DNA testing to guide therapeutic decisions in B-cell lymphomas. Curr Opin Hematol. 2019;26(4):281-7.

26. Hohaus S, Giachelia M, Massini G, et al. Cell-free circulating DNA in Hodgkin's and non-Hodgkin's lymphomas. Ann Oncol. 2009;20(8):1408-13.

27. Camus V, Stamatoullas A, Mareschal S, et al. Detection and prognostic value of recurrent exportin 1 mutations in tumor and cell-free circulating DNA of patients with classical Hodgkin lymphoma Haematologica. 2016; 101(9):1094-101.

28. Schwarzenbach H, Hoon DS, Pantel K. Cell-free nucleic acids as biomarkers in cancer patients. Nat Rev Cancer. 2011;11(6):426-37.

29. Bohers E, Viailly PJ, Becker S, et al. Non-invasive monitoring of diffuse large B-cell lymphoma by cellfree DNA high-throughput targeted sequencing: analysis of a prospective cohort. Blood Cancer $\mathrm{J}$. 2018;8(8):74.

30. Kurtz DM, Scherer F, Jin MC, et al. Circulating Tumor DNA Measurements As Early Outcome Predictors in Diffuse Large B-cell Lymphoma. J Clin Oncol. 2018;36(28):2845-53.

31. Scherer F, Kurtz DM, Newman AM, et al. Distinct biological subtypes and patterns of genome evolution in lymphoma revealed by circulating tumor DNA. Sci Transl Med. 2016;8(364):364ra155.

32. Rossi D, Diop F, Spaccarotella E, et al. Diffuse large B-cell lymphoma genotyping on the liquid biopsy. Blood. 2017;129(14):1947-57.

33. Assouline SE, Nielsen TH, Yu S, et al. Phase 2 study of panobinostat with or without rituximab in relapsed diffuse large B-cell lymphoma. Blood. 2016;128(2):185-94. 
34. Coccaro N, Anelli L, Zagaria A, Perrone T, Specchia G, Albano F. Molecular Complexity of Diffuse Large B-Cell Lymphoma: Can It Be a Roadmap for Precision Medicine? Cancers. 2020;12(1):185.

35. Kurtz DM. Prognostication with circulating tumor DNA: is it ready for prime time? Hematology Am Soc Hematol Educ Program. 2019; (1):47-52.

36. Rossi D, Kurtz DM, Roschewski M, Cavalli F, Zucca E, Wilson WH. The development of liquid biopsy for research and clinical practice in lymphomas: Report of the 15-ICML workshop on ctDNA. Hematol Oncol. 2020;38(1):34-7.

37. Camus V, Sarafan-Vasseur N, Bohers E, et al. Digital PCR for quantification of recurrent and potentially actionable somatic mutations in circulating free DNA from patients with diffuse large Bcell lymphoma. Leuk Lymphoma. 2016;57(9):2171-9.

38. Dubois S, Mareschal S, Picquenot JM, et al. Immunohistochemical and genomic profiles of diffuse large B-cell lymphomas: Implications for targeted EZH2 inhibitor therapy? Oncotarget. 2015;6(18):16712-24.

39. Morin RD, Johnson N, Severson TM, et al. Somatic mutations of EZH2 (Y641) in Follicular and Diffuse Large B-cell Lymphomas of Germinal Center Origin. Nat Genet. 2010;42(2):181-5.

40. Caganova M, Carrisi Ch, Varano G, et al. Germinal center dysregulation by histone methyltransferase EZH2 promotes lymphomagenesis. J Clin Invest. 2013;123(12):5009-22.

41. Zhang J, Dominguez-Sola D, Hussein S, et al. Disruption of KMT2D perturbs germinal center B cell development and promotes lymphomagenesis. Nat Med. 2015;21(10):1190-8.

42. Nagy Á, Bátai B, Balogh A, et al. Quantitative Analysis and Monitoring of EZH2 Mutations Using Liquid Biopsy in Follicular Lymphoma. Genes. 2020;11(7):785.

43. Tuaeva NO, Falzone L, Porozov YB, et al. Translational Application of Circulating DNA in Oncology: Review of the Last Decades Achievement. Cells. 2019;8(10):1251.

44. Statements \& Declarations.

\section{Figures}


Tumor Codon 641TAC wt

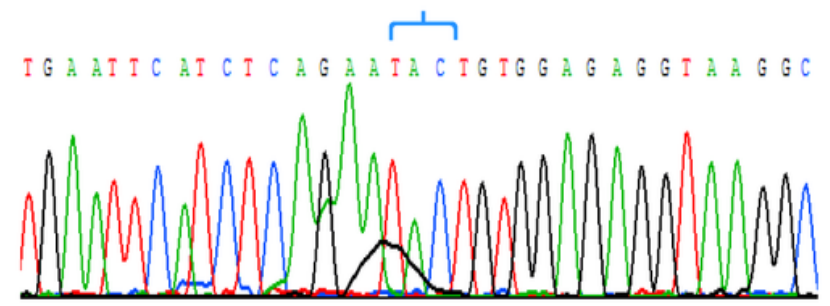

Tumor $\quad$ TAC>CAC $\mathrm{Y} 641 \mathrm{H}$

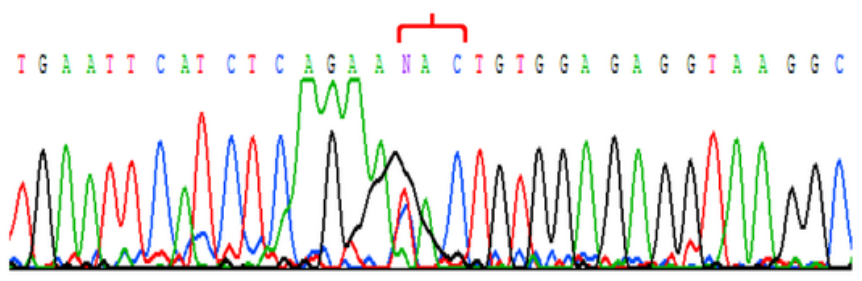

Plasma Codon 641TAC wt

TGAATCAICICAGAATACTGIGGAGAGGTAAGGC

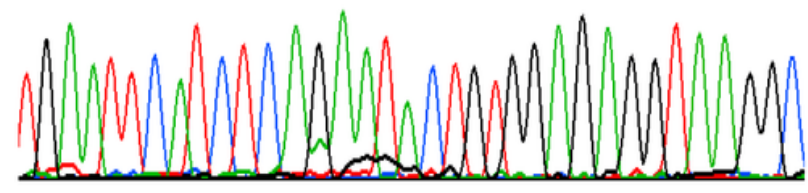

Plasma $\quad$ TAC>CAC $\mathrm{Y} 641 \mathrm{H}$

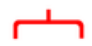

IGA AIICAICICAGA A IIACIGIGGAGAGGIA A G G C

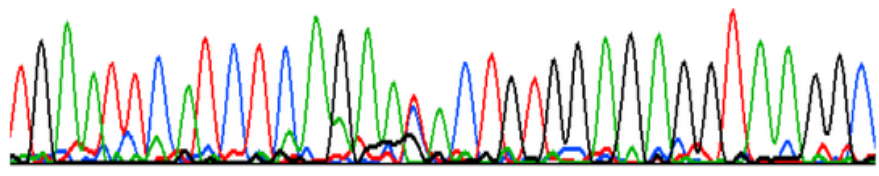

Figure 1

Chromatograms from Sanger sequencing.

A. Tumors and B. Plasma paired samples, with wildtype EZH2-Exon 16 and C. Tumor and D. Plasma paired samples with $E Z H 2$ mutated at codon $641(\mathrm{Y} 641 \mathrm{H})$. The wildtype sequence of codon 641 is TAC. 


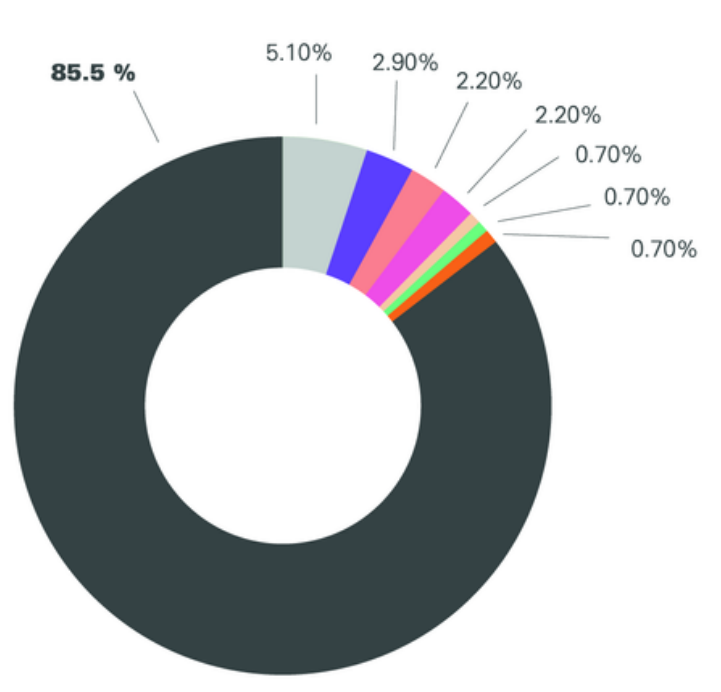

Sanger tumor

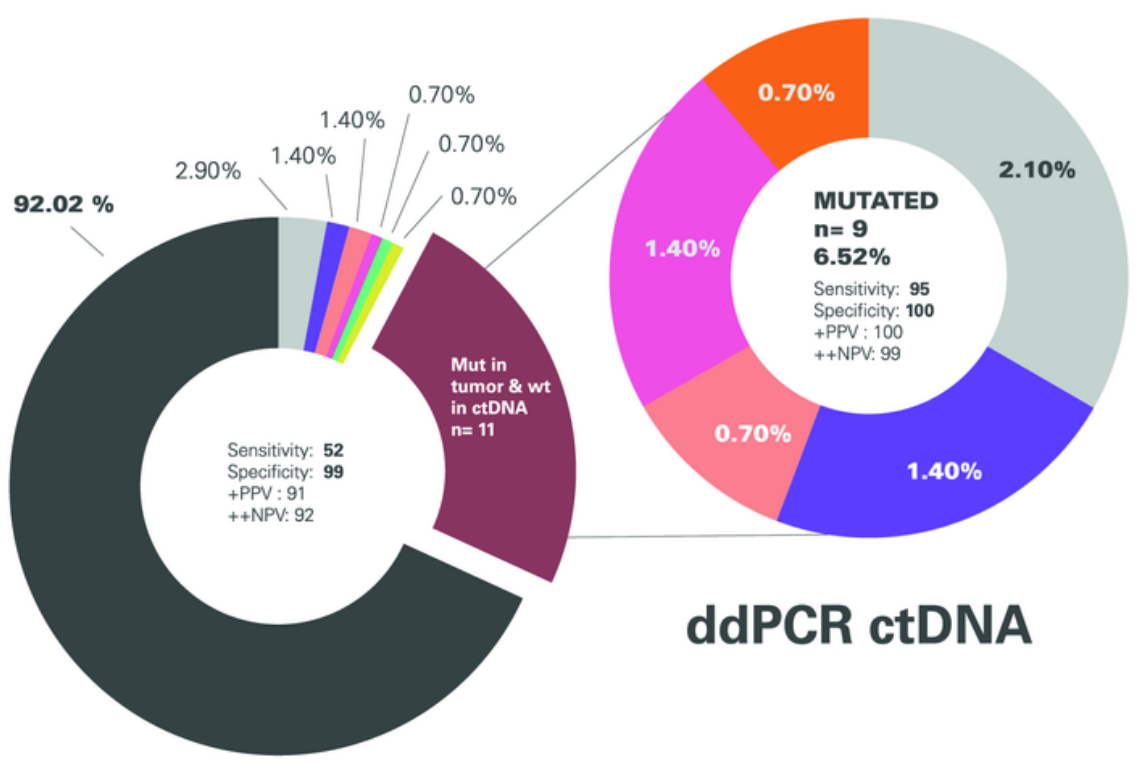

Sanger ctDNA

Y641F

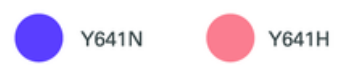

Y $641 \mathrm{~S}$

I638T

$Y 641 N+F 637 L$

$\mathrm{Y} 641 \mathrm{~S}+\mathrm{Y} 641 \mathrm{~F}$

Y641C

C $w$

\section{Figure 2}

Frequency of mutations in the tumor and ctDNA.

Tumor analysis was the standard of reference. ctDNA was initially analyzed by Sanger sequencing in all samples. Those cases in which the mutation was detected in the tumor samples but not in the plasma by Sanger sequencing, were evaluated by ddPCR.

+ PPV: Positive predictive value

++NPV: Negative predictive value 
A. Progression free-survival. Analysis in tumor DNA

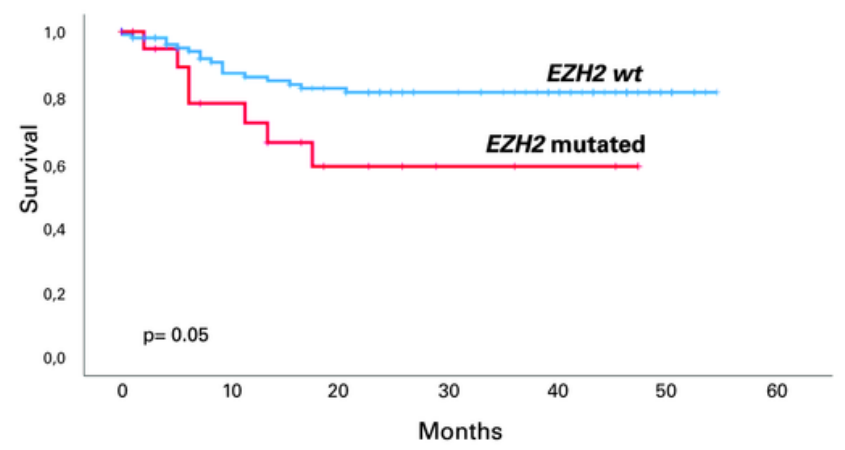

C. Risk of relapse. Analysis in tumor DNA

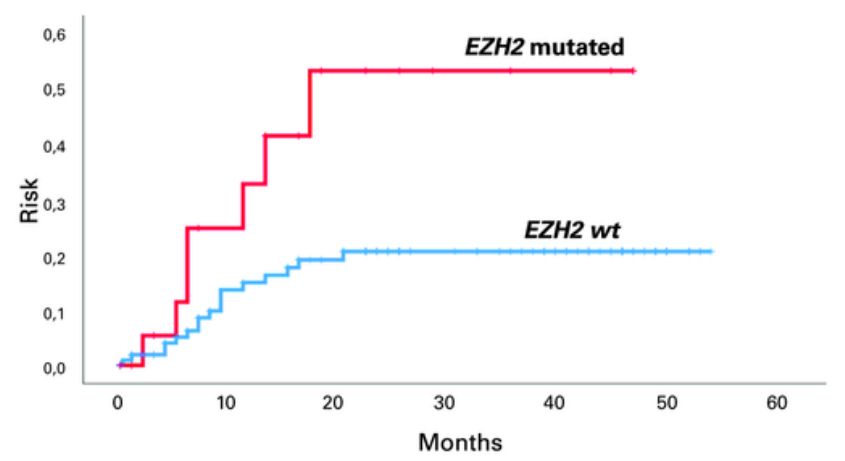

B. Progression free-survival. Analysis in ctDNA

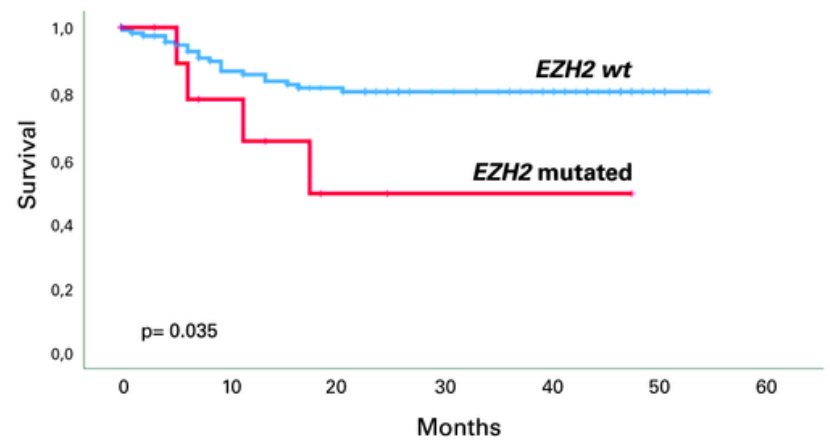

D. Risk of relapse. Analysis in ctDNA

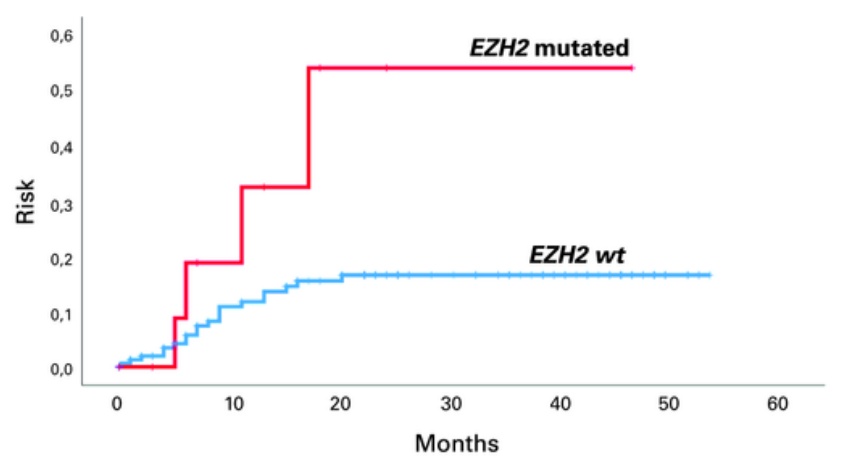

Figure 3

Progression free survival and risk of relapse curves, according to $E Z H 2$ mutation status.

Progression free survival in tumor (A) and ctDNA (B), and risk of relapse, when analyzing tumor (C) and ctDNA by ddPCR (D)

Blue line $=E Z H 2$ wildtype, Red line $=E Z H 2$ Mutated 